\title{
Prediction of quasi-one-dimensional superconductivity in metastable two-dimensional boron
}

\author{
Tong Chen, ${ }^{1}$ Qinyan Gu, ${ }^{1}$ Qun Chen, ${ }^{1}$ Xiaomeng Wang, ${ }^{1}$ Chris J. Pickard ${ }^{2,3}$ Richard J. Needs, ${ }^{4}$ \\ Dingyu Xing, ${ }^{1}$ and Jian Sun $\odot^{1, *}$ \\ ${ }^{1}$ National Laboratory of Solid State Microstructures, School of Physics and Collaborative Innovation Center of Advanced Microstructures, \\ Nanjing University, Nanjing, 210093, People's Republic of China \\ ${ }^{2}$ Department of Materials Science and Metallurgy, University of Cambridge, 27 Charles Babbage Road, \\ Cambridge CB3 OFS, United Kingdom \\ ${ }^{3}$ Advanced Institute for Materials Research, Tohoku University, 2-1-1 Katahira, Aoba, Sendai 980-8577, Japan \\ ${ }^{4}$ Theory of Condensed Matter Group, Cavendish Laboratory, J J Thomson Avenue, Cambridge CB3 OHE, United Kingdom
}

(Received 24 September 2019; revised manuscript received 19 January 2020; accepted 12 February 2020; published 27 February 2020)

\begin{abstract}
Two-dimensional (2D) boron has been predicted to exhibit various structural polymorphs and interesting physics, including superconductivity, ideal strength, negative Possion's ratio, and a higher thermal conductivity than graphene, etc. However, the difficulty in experimental synthesis of 2D boron has further reduced its exploration and applications. In this work we have used a crystal structure search method and first-principles calculations. We have proposed a scheme of "synthesize under high pressure and peel off at ambient conditions" to first predict a bulk phase of $\mathrm{KB}_{4}$, and then peel off free standing 2D boron from it. Based on electron-phonon coupling calculations, we estimated that $2 \mathrm{D}$ boron is an intrinsic superconductor with $T_{c}=17.9 \mathrm{~K}$. In addition we found a strong anisotropy of the electron-phonon coupling strength in $2 \mathrm{D}$ boron, thus the superconductivity in 2D boron possesses a quasi-1D feature. Our proposed scheme for the synthesis of free standing 2D boron will stimulate further studies of superconductivity in low dimensional materials, both theoretically and experimentally.
\end{abstract}

DOI: 10.1103/PhysRevB.101.054518

Two-dimensional (2D) superconductors have attracted much attention for their potential applications in nanoelectronics arising from their promising materials properties. Some of them, especially magic angle graphene, may potentially provide hints for understanding the mechanism of high-temperature superconductivity in these systems [1,2]. Monolayer transition metal dichalcogenides in which electrons are mainly restricted within a single layer, may exhibit 2D characteristics and some of them exhibit superconducting behavior. For instance, monolayer $\mathrm{NbSe}_{2}$ was reported to be superconducting with a critical temperature of $\left(T_{c}\right) \approx 8 \mathrm{~K}$ [3]. The superconducting $T_{c}$ of $2 \mathrm{D}$ superconductors can be tuned by doping, strain, gate voltage, and substrate, etc. For example, semiconducting layered $\mathrm{MoS}_{2}$ [4,5] and $\mathrm{WS}_{2}$ [6] can be engineered to be superconducting by doping and gating control. Monolayer FeSe on a STO substrate has a substantially higher $T_{c}$ than bulk FeSe [7]. Although intrinsic graphene has very weak electron-phonon coupling (EPC), due to its point-like Fermi surface, Li doped graphene can become superconducting [8]. With doping or strain, several elemental monolayers including phosphorene [9], arsenene [10], antimonene [11], and silicone [12] were predicted to be superconductors with a very low $T_{c}$ (several Kelvin). However, compared to the intrinsic superconductor, these additional tuning operations significantly complicate experimental realizations and make the systems difficult to control. Moreover,

*Corresponding author: jiansun@ nju.edu.cn heavy doping and large strain are difficult to realize in experiments and may even cause dynamical instabilities [11]. Thus, a free standing intrinsic layered superconductor would be ideal for both fundamental research and applications.

Elemental boron has complicated bonding characteristics, especially the multicenter bonds, including electron-deficient 1-electron 2-centers, 2-electrons 2-centers, 2-electron 3 -centers, etc. [13]. It also exhibits various crystal structures under different pressures and temperatures. [14] Due to the light atomic mass, boron has high Debye temperature, which makes boron and boron compounds possibly to have high superconducting $T_{c}$. On the other hand, due to the short and strong B-B covalence bonds, boron and boron compounds are also good candidates for superhard materials, such as $\mathrm{BN}$ [15], $\mathrm{BC}_{3}$ [16], and $\mathrm{B}_{6} \mathrm{C}$ [17], etc. Due to the similarity of boron and carbon, various allotropes of $2 \mathrm{D}$ boron with fascinating physical properties were predicted. For instance, similar to graphene, boron layers have been predicted to possess nontrivial topological Dirac cones [18-21]. 2D boron has also been predicted to have unusual mechanical properties (ideal strength, negative Possion's ratio [22]), and transport properties (higher thermal conductivity than graphene [23]). In addition, several structures of 2D boron have been predicted to be intrinsic phonon-mediated superconductors [24-26], whose critical temperature can be tuned by doping and application of strain [27]. However, the experimental realization of superconducting $2 \mathrm{D}$ boron is still very challenging, which obstructs further exploration. Up to now, only three allotropes of 2D boron have been synthesized, including an $\alpha$-sheet, $\beta-12$, and $\chi-3$. 
Unfortunately, superconductivity of 2D boron has not yet been observed, although the $\alpha$-sheet, $\beta-12$, and the $\chi-3$ sheet were predicted to be intrinsic superconductors. Recent studies have revealed that interference from the substrate, strain or doping could strongly suppress superconductivity of 2D boron [27]. Therefore, more effective synthesis methods are urgently required, especially methods for preparing free standing boron layers. A free standing superconducting 2D boron would be ideal for fabricating 2D superconducting devices, together with other 2D materials using layer-by-layer techniques.

In this work we followed a scheme of "synthesize under high pressure, peel off at ambient condition" [28] to search for new 2D boron allotropes, similar to intercalation compounds [29]. More specifically, we proposed the synthesis of bulk boron layer-intercalation compounds with alkali metals or alkaline earth metals via the high pressure method, then quench it to ambient conditions, and finally, peel off the 2D boron layer from its parent bulk phase, using electrochemical exfoliation or mechanical exfoliation methods. Using a crystal structure search method based on first-principles calculations, we have successfully found a $C 2 / m \mathrm{~KB}_{4}$ compound for such a purpose. All of the calculated formation enthalpies and energies, convex hulls and phonon spectra show that $\mathrm{KB}_{4}$ is metastable and could be synthesized experimentally. Our calculations also showed that the boron layer can be peeled off from $\mathrm{KB}_{4}$. Importantly, we predicted that the $2 \mathrm{D}$ boron synthesized by this method is a superconductor with a $T_{c}$ of $17.9 \mathrm{~K}$.

A crystal structure search was performed using a machine learning accelerated crystal structure search method based on Bayesian optimization [30], this method has been successfully applied in many systems, including helium-water compounds [31], metal pentazolate salts [32], and $\mathrm{Au}_{2} \mathrm{~Pb}$ [33], etc. Our results were also checked by the $a b$ initio random structure searching method [34] and the Cambridge Sequential Total Energy Package (CASTEP) plane-wave density-functional theory (DFT) code [35]. The firstprinciples calculations were performed using the Vienna $a b$ initio simulation package (VASP) [36] utilizing the generalized gradient approximation (GGA) and the Perdew-BurkeErnzerhof (PBE) exchange-correlation functionals [37]. Electron-phonon coupling constants were calculated using the QUANTUM-ESPRESSO code, the PBE exchange-correlation functional and norm-conserving pseudopotentials [38]. More technical details are provided in the Appendix.

After an extensive search, we found an excellent candidate, the $C 2 / m$ phase of $\mathrm{KB}_{4}$, which has a sandwich structure stacking with a potassium and a boron layer. The boron atoms form a double layer consisting of six-membered rings and the potassium atoms are located on top of the center of the six-membered rings, as shown in Fig. 1(a). The formation enthalpies relative to elemental potassium (fcc and bcc structures) and boron $\left(\mathrm{B}_{12}\right)$ are shown in Fig. 1(c) with a green line. The negative value of the formation enthalpy above $7 \mathrm{GPa}$ indicates that crystalline $\mathrm{KB}_{4}$ is thermodynamically stable at high pressure. Calculations with various different van der Waals interactions, including the DFT-D3 [39] and optB88-van der Waals (vdw) [40] functionals, yields similar results, as shown in Fig. 1(c). From the convex hull of K-B (a)

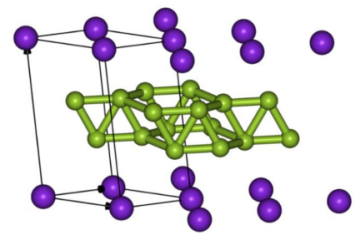

(c)
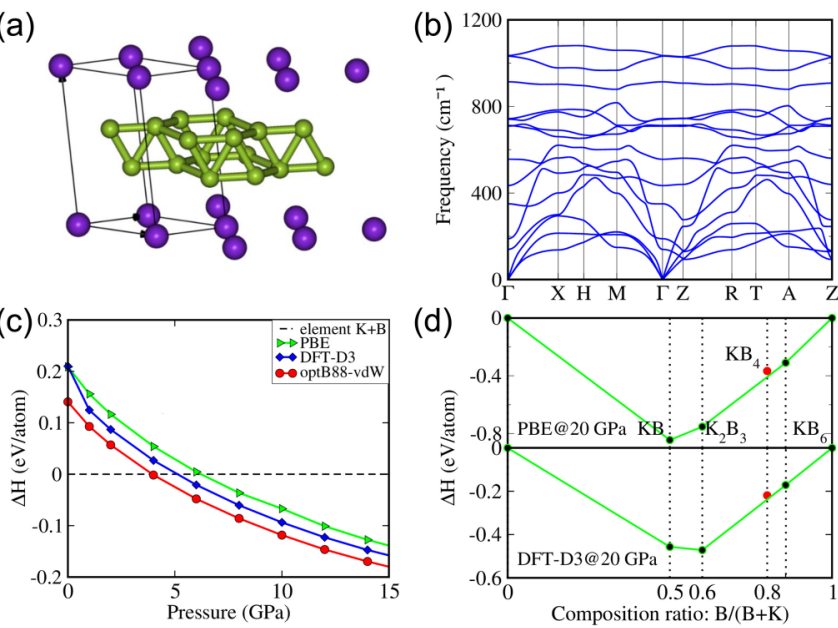

(d)

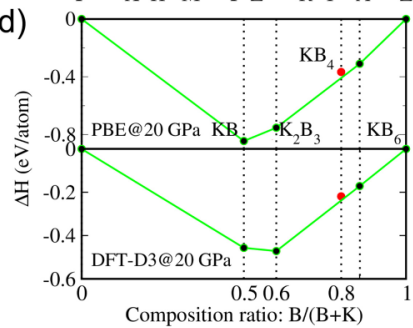

FIG. 1. (a) Side view of bulk $C 2 / m \mathrm{~KB}_{4}$. The potassium and boron atoms are represented with purple and green balls, respectively. (b) The phonon spectra of bulk $\mathrm{KB}_{4}$ at 20GPa. (c) The formation enthalpy relative to elemental potassium and boron, calculated with/without van der Waals interactions. (d) Convex hulls of the K-B system at $20 \mathrm{GPa}$ calculated with pure PBE [37] and DFT-D3 functionals [39].

system shown in Fig. 1(d), we find that the $C 2 / m \mathrm{~KB}_{4}$ phase is one of the metastable states of the K-B system at $20 \mathrm{GPa}$ (about $41 \mathrm{meV} /$ atom and $19 \mathrm{meV} /$ atom above the convex hull with PBE and DFT-D3-vdw, respectively. The convex hull calculated with optB88-vdw is also shown in the Appendix, Fig. 2). A large portion ( $20 \%)$ of the experimentally synthesized materials in the inorganic crystal database are actually metastable [41]. Many compounds with positive formation enthalpies are successfully synthesized with various techniques such as high pressure/high temperature, catalyzer and nonequilibrium conditions $[42,43]$. Therefore, predictions on metastable compounds are still meaningful. The dynamical stability of bulk $\mathrm{KB}_{4}$ at $20 \mathrm{GPa}$ is demonstrated by its phonon spectrum as shown in Fig. 1(b), without an imaginary frequency. Its phonon spectra indicate that it is stable even at ambient pressure, as shown in the Appendix, Fig. 3 and therefore crystalline $\mathrm{KB}_{4}$ should be quenchable to ambient conditions. We also found candidate $\mathrm{KB}$ structures with space group $P 6_{3} / m m c, \mathrm{~K}_{2} \mathrm{~B}_{3}$ with space group Cmce, and $\mathrm{KB}_{6}$ with space group $P m-3 m$. Detailed information on these phase is shown in the Appendix Figs. 4-6, and Table I.

We then investigated the electronic structures of the $\mathrm{KB}_{4}$ phase. As shown by the electronic band structures and orbital

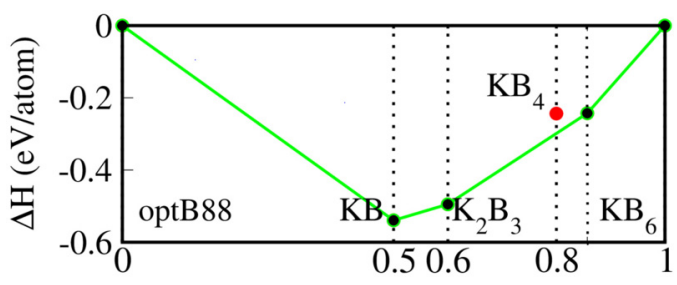

FIG. 2. The Convex hulls of the K-B system at $20 \mathrm{GPa}$ calculated with optB88-vdw methods. The $\mathrm{KB}_{4}$ is about $55 \mathrm{meV} /$ atom higher than the convex hull. 


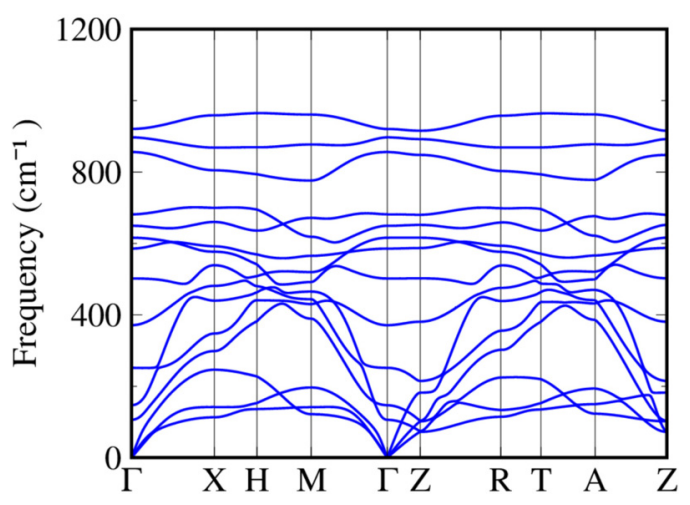

FIG. 3. The phonon spectrum of $\mathrm{KB}_{4}$ at ambient condition.

projected density of states (DOS) of $\mathrm{KB}_{4}$ at $20 \mathrm{GPa}$ in Fig. 7(a), $\mathrm{KB}_{4}$ has a metallic character and the $\mathrm{B}-p_{y}$ and B- $p_{z}$ orbitals contribute the major contribution to the total DOS at the Fermi level. Two bands cross the Fermi level and form several Fermi pockets within the Brillouin zone (BZ). As shown in Fig. 7(b), one large electron pocket passes through the entire $\mathrm{BZ}$ along the $k_{z}$ direction and encloses the $\Gamma$ and $\mathrm{Z}$ points, together with several small hole pockets marked with a cyan color. As we proposed, once $\mathrm{KB}_{4}$ is synthesized and quenched to ambient condition, we can adopt the electrochemical exfoliation method to prepare 2D boron samples. To evaluate the feasibility of exfoliation, we calculate the exfoliation energy, as shown in Fig. 7(c). The calculated exfoliation energy of $2 \mathrm{D}$ boron obtained directly from bulk $\mathrm{KB}_{4}$ is about $150 \mathrm{meV} / \AA^{2}$ and thus the boron layer may potentially be exfoliated. As an alternative method, it is also possible to obtain pure boron layers by evaporating potassium atoms from $\mathrm{KB}_{4}$, using a similar method as in the synthesis of $\mathrm{Si}_{24}$ from $\mathrm{Na}_{4} \mathrm{Si}_{24}[44,45]$ and synthesis of $2 \mathrm{D} \mathrm{ZnSb}$ from selective etching of $\mathrm{Li}$ ions from $\mathrm{LiZnSb}$ with polar solvent solution reaction [46]. Figure 7(d) shows the calculated energy barriers for a potassium atom diffusing in $\mathrm{KB}_{4}$, along two different diffusion paths. The potential energy barrier is around $0.31 \mathrm{eV}$, which is much smaller than that of sodium atoms diffusing in $\mathrm{Na}_{4} \mathrm{Si}_{24}$ (around $1.7 \mathrm{eV}$ ) as calculated in Ref. [28], and this (a)

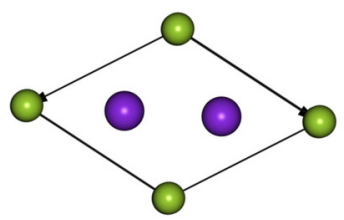

(c)

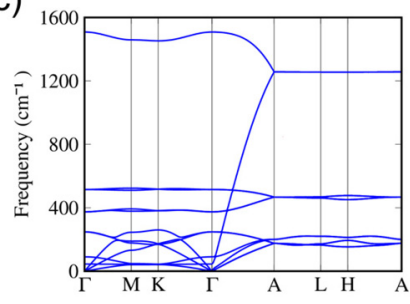

(b)

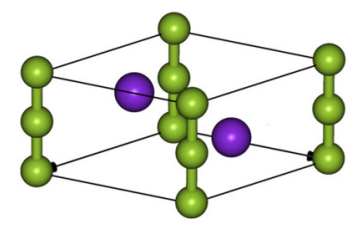

(d)

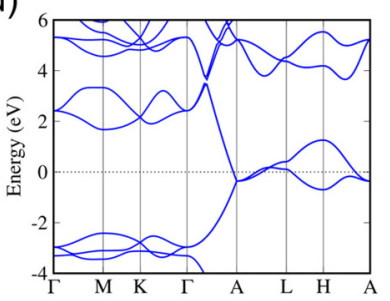

FIG. 4. The top (a) and side (b) view of the KB crystal. Phonon spectra (c) and band structures (d) of candidate structure KB at $25 \mathrm{GPa}$. (a)

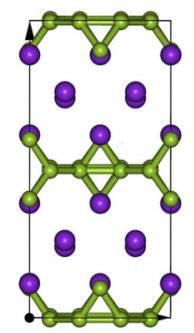

(b)

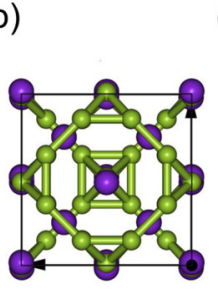

(c)

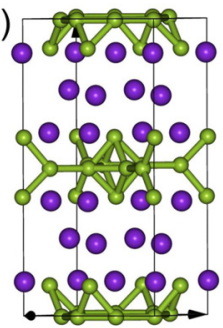

(d)
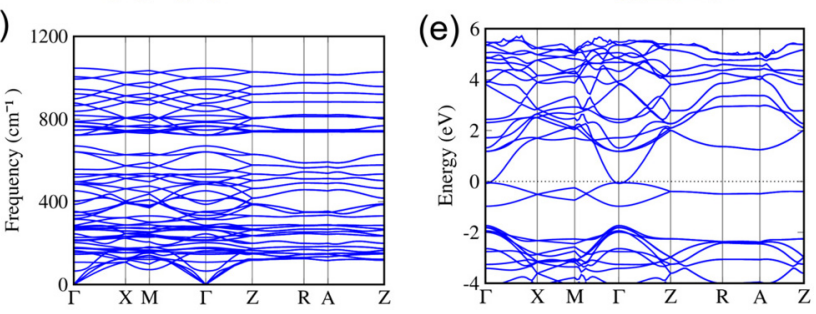

FIG. 5. The (a) front, (b) top and (c) side view of the $\mathrm{K}_{2} \mathrm{~B}_{3}$ crystal. Phonon spectra (d) and band structures (e) of candidate structure $\mathrm{K}_{2} \mathrm{~B}_{3}$ at $25 \mathrm{GPa}$.

indicates that the potassium atoms should readily evaporate. If the potassium atoms are absent, the exfoliation energy of 2D boron from pure boron layers almost vanishes at about $0.4 \mathrm{meV} / \AA^{2}$, as shown in Fig. 7(c) with a green circle dotted line. This relatively low exfoliation energy stems from the fact that the boron electrons tend to localize within the stacked boron double layer rather than the interlayer space. Thus there are essentially no interaction between the layers, which makes it fairly easy to peel off the 2D boron from the bulk boron layers.

To understand the high exfoliation energy of $2 \mathrm{D}$ boron from bulk $\mathrm{KB}_{4}$ we performed a Bader charge analysis, which shows that around 0.6 electrons transfer from one potassium atom to the boron layers. The electron localization function $(E L F)$ (Isosurface level $=0.36$ ) also verified this result qualitatively. As plotted in Fig. 8(a), most of the electrons are localized in boron layers, while very few electrons remain within the vicinity of the potassium atoms. There is no electron localization between the boron layer and the potassium layer, (a)

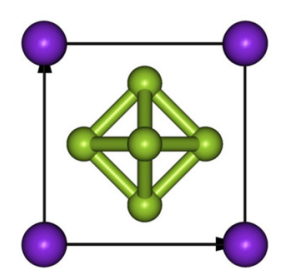

(c)

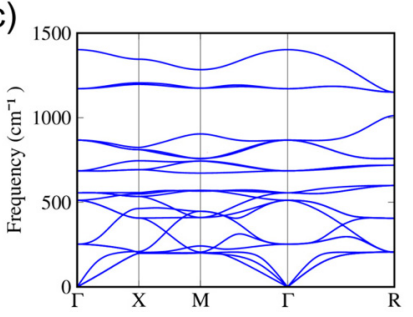

(b)

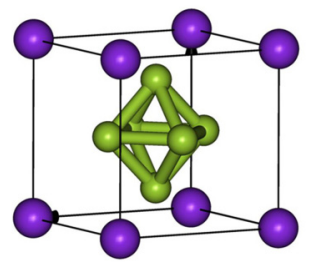

(d)

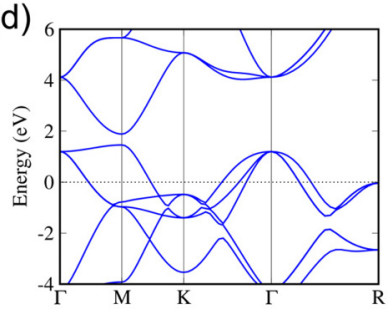

FIG. 6. The (a) top, (b) side view of the $\mathrm{KB}_{6}$ crystal. Phonon spectra (c) and band structures (d) of candidate structure $\mathrm{KB}_{6}$ at $25 \mathrm{GPa}$ 
TABLE I. The details of the crystal structures of the $\mathrm{KB}, \mathrm{K}_{2} \mathrm{~B}_{3}$ and $\mathrm{KB}_{6}$ at $25 \mathrm{GPa}$.

\begin{tabular}{lccc}
\hline \hline Phase & Lattice parameter $(\AA)$ & Wyckoff position & Atomic coordinates (Fractional) \\
\hline Bulk KB phase $\left(P 6_{3} / m m c\right)$ & $a=4.6553$ & $\mathrm{~K}(2 b)$ & $0.33333,0.66667,0.25000$ \\
& $c=3.0280$ & $\mathrm{~B} 1(2 a)$ & $0.00000,0.00000,0.50000$ \\
Bulk K ${ }_{2}$ phase $(\mathrm{Cmce})$ & $a=5.9995$ & $\mathrm{~K} 1(8 f)$ & $0.50000,0.11500,0.48356$ \\
& $b=12.6266$ & $\mathrm{~K} 5(8 e)$ & $0.25000,0.23967,0.25000$ \\
& $c=6.0297$ & $\mathrm{~B} 1(16 g)$ & $0.64525,0.00464,0.14488$ \\
Bulk KB 6 phase $(P m-3 m)$ & $a=4.0259$ & $\mathrm{~B} 9(8 f)$ & $0.50000,0.10017,0.01312$ \\
& & $\mathrm{~K} 1(1 a)$ & $0.00000,0.00000,0.00000$ \\
& & $\mathrm{~B} 1(6 f)$ & $0.19724,0.50000,0.50000$ \\
\hline \hline
\end{tabular}

and we therefore infer that only a few of them have ionic-like interactions among them. With the help of the reduced density gradient (RDG) method proposed in Ref. [47], we study the interlayer noncovalent interactions. In Fig. 8(b) we plot the electron density multiplied by the sign of the second Hessian eigenvalue and its RDG for $\mathrm{KB}_{4}$. According to the theory, the spikes located at low-densities and low-gradient regions represent the week interaction. Figures 8(c) and 8(d) show side and top views of the gradient isosurfaces. The isosurfaces are colored with a blue-green-red scale on referring to values of $-\operatorname{sign}\left(\lambda_{2}\right) \rho$, where a green color indicates the week interactions. In Fig. 8(c), the green section, which is subject to the boron and potassium layers, corresponds to the spikes in Fig. 8(b). These green gradient isosurfaces provide a vivid visualization of the interactions in real space. From the top view of Fig. 8(d), we can see that the green isosurfaces form an elliptical ring embedded between the six-membered boron ring and potassium atom.

The dynamical stability of 2D boron can be confirmed by its phonon dispersion in Fig. 9(e), with the absence of imaginary frequencies. 2D boron belongs to space group $C 2 / \mathrm{m}$. It forms a double layer stacking of two six-membered boron (a)

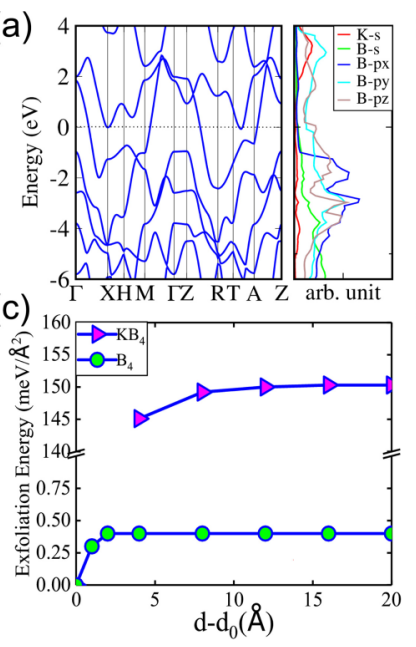

(b)

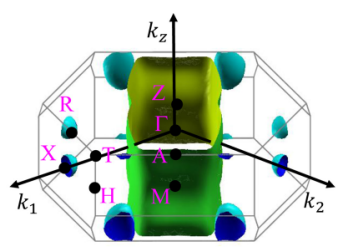

(d)

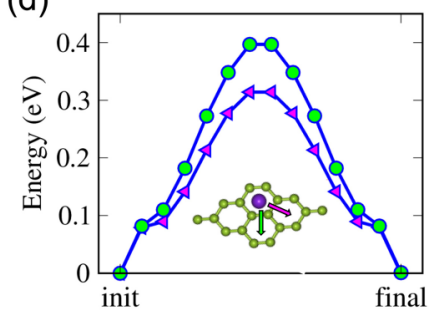

FIG. 7. (a) The band structures and DOS of $\mathrm{KB}_{4}$ at $20 \mathrm{GPa}$. (b) Fermi surfaces of $\mathrm{KB}_{4}$. (c) Calculated exfoliation energy of a $2 \mathrm{D}$ boron layer from bulk $\mathrm{KB}_{4}$ and bulk $\mathrm{B}_{4}$. (d) Energy barriers of potassium atoms to diffuse in $\mathrm{KB}_{4}$, the arrows in the inset represent the diffusion paths. rings, with stacking direction $-(\vec{x}+\vec{y})$, as shown in Fig. 9(a). The vectors enclose the primitive cell with four atoms. The six-membered ring does not provide a perfect honeycomb-like structure, because the bonds along the zigzag direction are of length $1.774 \AA$. This is a little longer than the length in the armchair direction $(1.722 \AA)$, which may explain why the energy barriers along the two pathways marked in Fig. 7(d) differ. Details of the crystal structure information for bulk $\mathrm{KB}_{4}$ and the 2D boron layer are listed in Table II. To double check the stability of the $\mathrm{KB}_{4}$ and $2 \mathrm{D}$ boron, we perform $a b$ initio molecular dynamic simulations at room temperature based on the ambient pressure structures. And no structure collapse has been observed after 10 ps, results are shown in Appendix Fig. 10.

Figure 9(b) shows projected band structures and the DOS of a $2 \mathrm{D}$ boron layer. Compared with $\mathrm{KB}_{4}$, we find that their band structures are quite similar in general, except at the Fermi level. Without the doping effects arising from the potassium atoms, the Fermi level of the boron layer drops. As for the 2D boron layer, the $s$ orbital is far away from the Fermi level and the $p_{x}$ orbitals are mainly found within the energy range -4 to $-2 \mathrm{eV}$. Only the $p_{y}$ and $p_{z}$ orbitals participate (a)

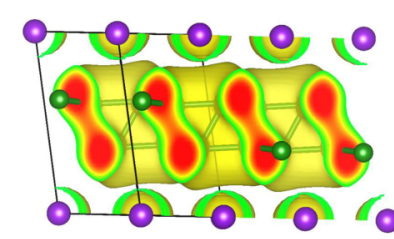

(c)

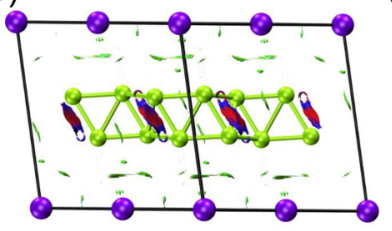

(b)

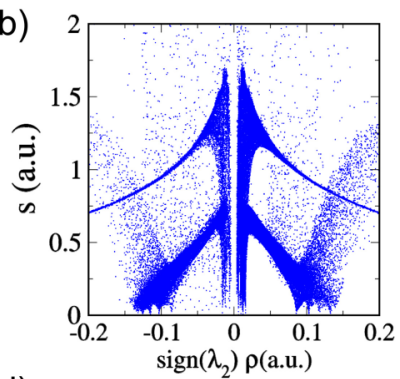

(d)

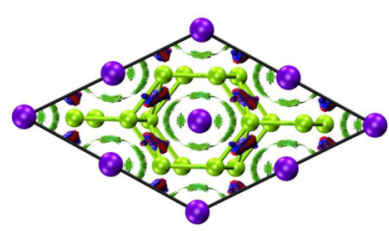

FIG. 8. (a) The ELF of $\mathrm{KB}_{4}$. (b) Plots of the electron density multiplied by the sign of the second Hessian eigenvalue and its RDG for $\mathrm{KB}_{4}$. (c) Side view and (d) top view of the gradient isosurfaces of noncovalent interations. The surfaces are colored with a blue-greenred scale referring to values of $-\operatorname{sign}\left(\lambda_{2}\right) \rho$. Green indicates the very weak interaction. 


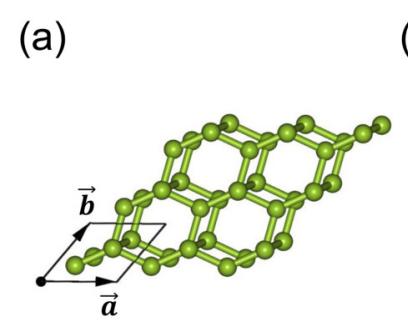

(d)

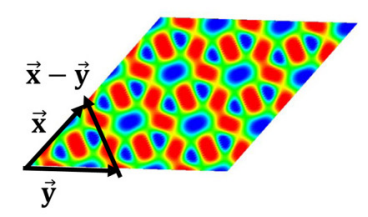

(b)

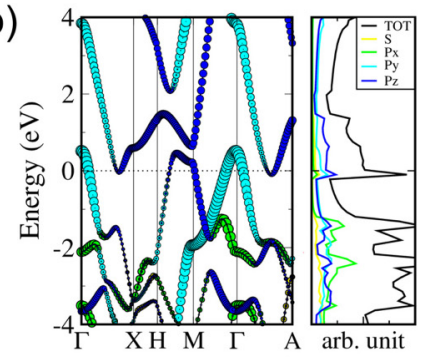

(e) 1

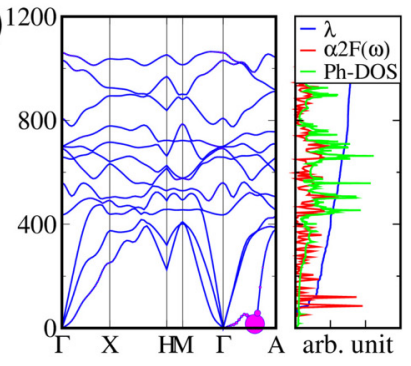

(c)

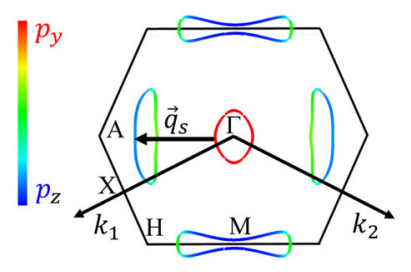

(f)

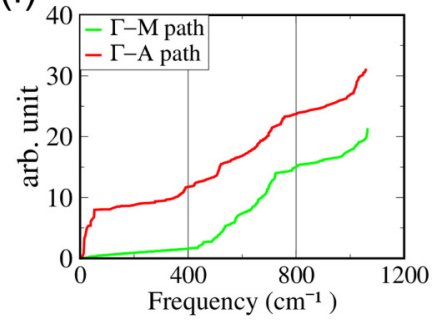

FIG. 9. (a) The crystal structure of a 2D boron layer. (b) The projected band structures and DOS. The orbital characters of the bands are depicted by the thickness and color. (c) $p_{y}$ and $p_{z}$ orbitals projected Fermi surface. (d) ELF at the (001) plane passing through the six-membered ring. (e) Phonon spectra (left) and Eliashberg spectral function $\alpha^{2} F(\omega)$, acumulated EPC strength $\lambda(\omega)$ and phonon DOS (right), the radium of the magenta circles in the phonon spectra represent the contribution to the EPC of the vibration mode. (f) EPC strength projected on different paths.

in the formation of the Fermi surface. Three bands cross the Fermi level and form three Fermi pockets in the BZ. Two electron pockets enclose the $\Gamma$ and $M$ points, respectively, and one hole pocket lies on the $\Gamma$-A path, as shown in Fig. 9(c). Figure 9(c) also displays the $p_{y}$ and $p_{z}$ orbitals projected onto the Fermi surface. Two electron pockets arise largely from the $p_{y}$ and $p_{z}$ orbitals. The hole pockets and their two components have approximately equal weights. Considering that the GGAPBE density functional always underestimates the band gap, we have also used a nonlocal hybrid PBE0-density functional and Tran-Blaha modified Becke-Johnson exact-exchange (xc) potential to double check the size of the band gap. The results show that the system remains metallic. The band structures and DOS are shown in the Appendix, Figs. 11 and 12.

Afterwards we studied the superconducting properties of 2D boron using electron-phonon coupling calculations with the GGA-PBE functionals. The superconducting critical temperature $\left(T_{c}\right)$ is estimated using the modified Allen-Dynes modified McMillan equation [48]: $T_{c}=$ $\frac{\omega_{\log }}{1.2} \exp \left[\frac{-1.04(1+\lambda)}{\lambda\left(1-0.62 \mu^{*}\right)-\mu^{*}}\right]$, where $\lambda, \alpha^{2} F(\omega)$ and $\omega_{\log }$ represent the EPC strength, the Eliashberg electron-phonon spectral function, and the logarithmically averaged phonon frequency, respectively. For this $2 \mathrm{D}$ boron layer, our theoretical analysis provides the estimation of $\lambda=0.827, \omega_{\log }=356.4 \mathrm{~K}, T_{c}=$ $17.9 \mathrm{~K}$ with a commonly used Coloumb repulsion coefficient of $\mu^{*}=0.1$.

On the right hand side of Fig. 9(e) we show a plot of the accumulated EPC strength $\lambda$. It is obvious that acoustical modes at low frequencies contribute more than half of the total magnitude of $\lambda$. Although there are also some peaks among the middle and high frequency regions, the contribution of these modes to the EPC strength are small because $\lambda$ is proportional to $\frac{1}{\omega}$. In the left side of Fig. 9(e), there is an obvious phonon softening along the $\Gamma$-A path (marked with a magenta circle), we have defined it as $\boldsymbol{q}_{s}, \boldsymbol{q}_{s}=(0.18,-0.18)$. This phonon dip characterizes a large contribution to the EPC. The Kohn anomaly is caused by interpocket Fermi surface nesting, as shown in Fig. 9(c). From the $p_{y}$ and $p_{z}$ orbitalprojected Fermi surface in Fig. 9(c), we find that the $\boldsymbol{q}_{\boldsymbol{s}}$ soft phonon vector along the $\Gamma-A$ direction helps to form Cooper pairs from the $p_{y}$ orbital in the hole pocket near the $\Gamma$ point and the $p_{z}$ orbital in the electron pocket.

Besides the soft phonon vector $\boldsymbol{q}_{s}$, we find that the contribution from the entire longitudinal acoustic mode to the

TABLE II. The crystal structure information of the $\mathrm{KB}_{4}$ and $2 \mathrm{D}$ boron layer.

\begin{tabular}{|c|c|c|c|}
\hline Phase & Lattice parameter $(\AA)$ & Wyckoff position & Atomic coordinates (Fractional) \\
\hline Bulk $\mathrm{KB}_{4}$ phase $(C 2 / m)$ & $\begin{array}{c}a=5.727 \\
b=2.936 \\
c=8.095 \\
\beta=126.315 \mathrm{deg}\end{array}$ & $\begin{array}{l}\mathrm{K}(2 a) \\
\mathrm{B} 1(4 i) \\
\mathrm{B} 2(4 i)\end{array}$ & $\begin{array}{l}0.00000,0.00000,0.00000 \\
0.68519,0.00000,0.39795 \\
0.02672,0.00000,0.62031\end{array}$ \\
\hline $2 \mathrm{D}$ boron layer $(C 2 / m)$ & $\begin{array}{c}a=5.721 \\
b=2.689 \\
c=20.535 \\
\beta=104.637 \mathrm{deg}\end{array}$ & $\begin{array}{l}\mathrm{B} 1(4 i) \\
\mathrm{B} 3(4 i)\end{array}$ & $\begin{array}{l}0.25646,0.00000,0.53186 \\
0.03573,0.00000,0.45997\end{array}$ \\
\hline
\end{tabular}




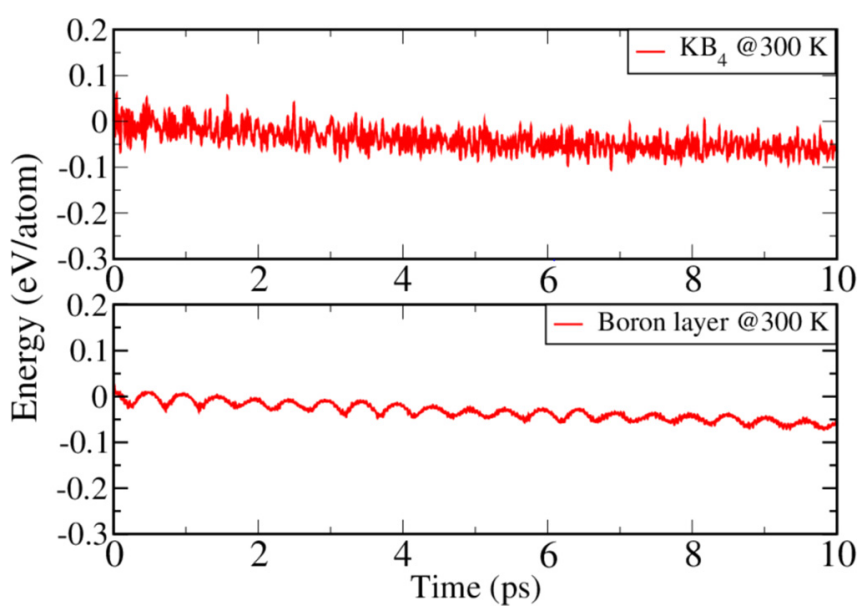

FIG. 10. The ab initio molecular dynamics simulations of bulk $\mathrm{KB}_{4}$ (top) and 2D boron layer (bottom) at $300 \mathrm{~K}$.

EPC along the $\Gamma-A$ path is considerably larger than that of the other paths, displayed in Fig. 9(e). In Fig. 9(f), we project the EPC strength to the $\Gamma-A$ and $\Gamma-M$ paths. The total strength of the EPC along the $\Gamma-A$ path is around 1.5 times that along the $\Gamma-M$ path. Remarkably, the accumulated strength of the path $\Gamma-A$ in the low frequency range (below $400 \mathrm{~cm}^{-1}$ ) is almost six times higher than that of for $\Gamma-M$ path. This shows strong anisotropic superconductivity in the $2 \mathrm{D}$ boron layer. In order to understand the underlying mechanisms, we investigate the electron distributions of the $2 \mathrm{D}$ boron layer from the ELF to analyze the transport properties. As the ELF of the boron layer in Fig. 9(d) shows, the cut plotted through the isosurface demonstrates that there is strong anisotropy in the electron distribution. The distribution along the zigzag direction is dense and continuous, due to the strong covalent bonding and lattice distortion along the zigzag direction; while that along the armchair direction contains several gaps between the boron atoms. The whole distribution depicts an anisotropic and quasi-1D character. In general, high electron density implies a greater probability of forming

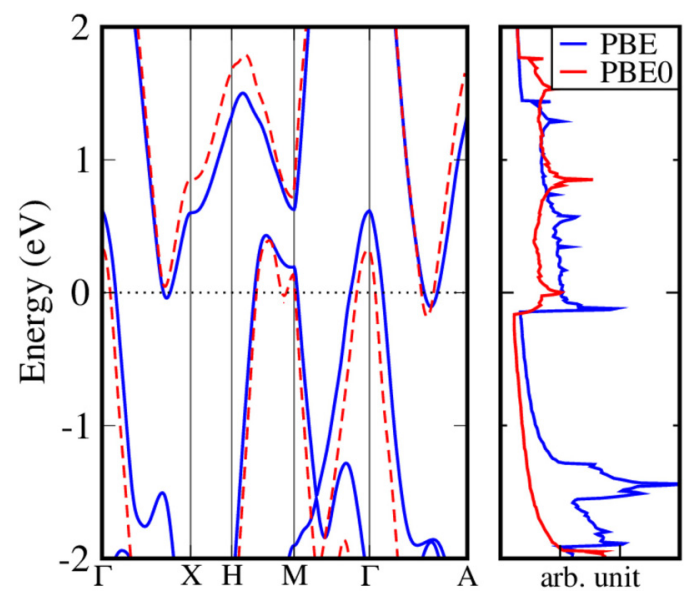

FIG. 11. Band structures and DOS of boron layer checked with nonlocal hybrid functional PBE0.

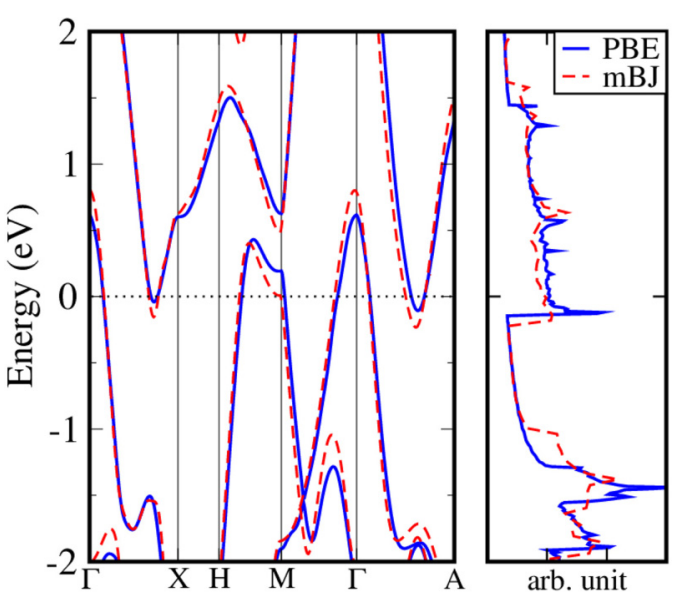

FIG. 12. Band structures and DOS of boron layer checked with Tran-Blaha modified Becke-Johnson xc potential. The results show that the system remain metallic. And the change of the DOS is tiny at the Fermi level.

Cooper pairs. This may be the underlying reason for the 1D-like superconductivity in the $2 \mathrm{D}$ boron layer.

Superconductivity within the boron layer is challenging for experimental observations since the influence from the substrate is difficult to eliminate. If free standing 2D boron could be synthesized using our method proposed above, it will facilitate the observation of superconductivity within the boron sheet. In addition, although the Fermi level is located slightly above the Van Hove singularity in our $C 2 / m 2 \mathrm{D}$ boron system, this still gives a $T_{c}$ of $17.9 \mathrm{~K}$. It may be possible to enhance the superconducting temperature if the Fermi level can be tuned to being closer to the Van Hove singularity, with strain, doping, or gate voltage.

In conclusion, we propose that a free standing superconducting $2 \mathrm{D}$ boron layer can be synthesized using a two-step method. The first step is to synthesize bulk $C 2 / m \mathrm{~KB}_{4}$ using the high pressure method. The second step is to quench bulk $\mathrm{KB}_{4}$ to ambient conditions, and then peel off a monolayer boron sheet from $\mathrm{KB}_{4}$ or pure $\mathrm{B}_{4}$, where the bulk $\mathrm{B}_{4}$ can be obtained from $\mathrm{KB}_{4}$ by evaporation of the potassium atoms. All of our results are from first-principles calculations, including the formation enthalpy, convex hull, phonon spectra of $C 2 / m \mathrm{~KB}_{4}$, and the $2 \mathrm{D}$ boron sheet. The exfoliation energies that we have proposed are both practical and feasible. Based on electron-phonon coupling theory, the calculated $T_{c}$ of the $2 \mathrm{D}$ boron layer is about $17.9 \mathrm{~K}$. The orbital projected band structure and Fermi surface have shown that the Cooper pairs are mainly formed from $p_{y}$ and $p_{z}$ orbitals. The interpocket Fermi nesting gives rise to a Kohn anomaly along the $\Gamma-A$ path, and the Kohn anomaly also provides a large contribution to the EPC constant. The path projected EPC strength reveals a unique anisotropy in the superconducting with a quasi-1D feature, which has not been found previously in 2D materials. Our free standing 2D boron layer will promote the study of superconductivity in low dimensional systems both in theory and experiments.

We thank H. Gao, J. Yuan, and K. Xia for valuable discussions. We gratefully acknowledge the financial support 
from the MOST of China (Grant No. 2016YFA0300404), the National Natural Science Foundation of China (Grants No. 11974162 and No. 11834006), and the Fundamental Research Funds for the Central Universities. The calculations were carried out using supercomputers at the High Performance Computing Center of Collaborative Innovation Center of Advanced Microstructures, the high-performance supercomputing center of Nanjing University and "Tianhe-2" at NSCC-Guangzhou.

\section{APPENDIX: METHODS}

The structural optimizing and self-consistent total energy calculations were performed with VASP [36] utilizing the GGA in the PBE exchange-correlation functionals [37]. The crystal structures are optimized with kinetic energy for plane-wave basis cutoff of $440 \mathrm{eV}$ and a $\Gamma$ centered $k$-points mesh with spacing of $2 \pi \times 0.025 \AA^{-1}$. The enthalpies was cross checked with DFT-D3 [39] and optB88-vdw [40] corrections. The band structures were checked by nonlocal hybrid functional PBE0 [49] with Wannier interpolation [50] and Tran-Blaha modified Becke-Johnson xc-potential [51]. Energy barriers of potassium diffusion were explored with the variable-cell nudged elastic band method, implemented in USPEX [52]. Phonon spectra were calculated with the PHONOPY package [53]. Noncovalent interactions were identified with the CRITIC2 package [54]. Electron-phonon interaction coefficient were calculated with the QUANTUM-ESPRESSO code utilizing PBE exchange-correlation functionals and normconserving pseudopotentials [38]. The kinetic energy cutoff
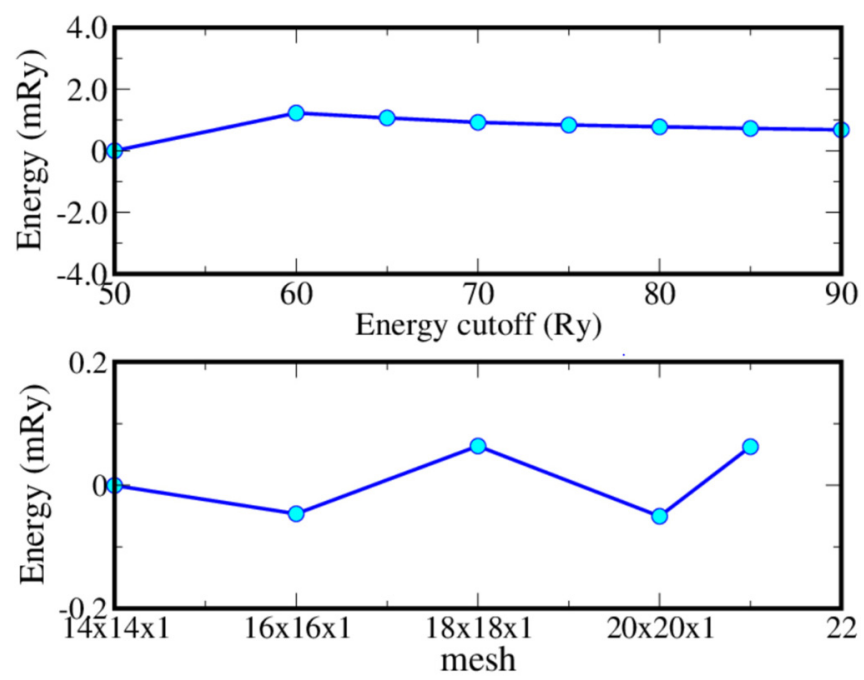

FIG. 13. The tests of kinetic energy cutoff (top) and $k$-points mesh (bottom)

and the charge density cutoff of the plane wave basis were chosen to be 80 and $320 \mathrm{Ry}$, respectively. The $k$-points mesh and kinetic energy cutoff tests are shown in Fig. 13. We evaluated the self-consistent total energy on a $20 \times 20 \times$ 1 Monkhorst-Pack grid (with spacing of $2 \pi \times 0.021 \AA^{-1}$ ). The electron-phonon interaction coefficients were executed in the framework of density-functional perturbation theory. We adopted a $60 \times 60 \times 1$ fine $k$-points grid for EPC linewidth integration and $10 \times 10 \times 1 q$-points grid for evaluating the dynamical matrix.
[1] Y. Saito, T. Nojima, and Y. Iwasa, Nat. Rev. Mater. 2, 16094 (2016).

[2] Y. Cao, V. Fatemi, S. Fang, K. Watanabe, T. Taniguchi, E. Kaxiras, and P. Jarillo-Herrero, Nature (London) 556, 43 (2018).

[3] X. Xi, Z. Wang, W. Zhao, J.-H. Park, K. T. Law, H. Berger, L. Forró, J. Shan, and K. F. Mak, Nat. Phys. 12, 139 (2015).

[4] J. T. Ye, Y. J. Zhang, R. Akashi, M. S. Bahramy, R. Arita, and Y. Iwasa, Science 338, 1193 (2012).

[5] J. M. Lu, O. Zheliuk, I. Leermakers, N. F. Q. Yuan, U. Zeitler, K. T. Law, and J. T. Ye, Science 350, 1353 (2015).

[6] J. Lu, O. Zheliuk, Q. Chen, I. Leermakers, N. E. Hussey, U. Zeitler, and J. Ye, Proc. Natl. Acad. Sci. USA 115, 3551 (2018).

[7] Q.-Y. Wang, Z. Li, W.-H. Zhang, Z.-C. Zhang, J.-S. Zhang, W. Li, H. Ding, Y.-B. Ou, P. Deng, and K. Chang et al., Chin. Phys. Lett. 29, 037402 (2012).

[8] B. M. Ludbrook, G. Levy, P. Nigge, M. Zonno, M. Schneider, D. J. Dvorak, C. N. Veenstra, S. Zhdanovich, D. Wong, and P. Dosanjh et al., Proc. Natl. Acad. Sci. USA 112, 11795 (2015).

[9] Y. Ge, W. Wan, F. Yang, and Y. Yao, New J. Phys. 17, 035008 (2015)

[10] X. Kong, M. Gao, X.-W. Yan, Z.-Y. Lu, and T. Xiang, Chin. Phys. B 27, 046301 (2018).

[11] A. V. Lugovskoi, M. I. Katsnelson, and A. N. Rudenko, Phys. Rev. B 99, 064513 (2019).

[12] W. Wan, Y. Ge, F. Yang, and Y. Yao, EPL 104, 36001 (2013).
[13] S. Mondal, S. van Smaalen, A. Schonleber, Y. Filinchuk, D. Chernyshov, S. I. Simak, A. S. Mikhaylushkin, I. A. Abrikosov, E. Zarechnaya, and L. Dubrovinsky et al., Phys. Rev. Lett. 106, 215502 (2011).

[14] A. R. Oganov, J. Chen, C. Gatti, Y. Ma, Y. Ma, C. W. Glass, Z. Liu, T. Yu, O. O. Kurakevych, and V. L. Solozhenko, Nature (London) 457, 863 (2009).

[15] Y. Tian, B. Xu, D. Yu, Y. Ma, Y. Wang, Y. Jiang, W. Hu, C. Tang, Y. Gao, and K. Luo et al., Nature (London) 493, 385 (2013).

[16] M. Zhang, H. Liu, Q. Li, B. Gao, Y. Wang, H. Li, C. Chen, and Y. Ma, Phys. Rev. Lett. 114, 015502 (2015).

[17] K. Xia, M. Ma, C. Liu, H. Gao, Q. Chen, J. He, J. Sun, H.-T. Wang, Y. Tian, and D. Xing, Mater. Today Phys. 3, 76 (2017).

[18] X.-F. Zhou, X. Dong, A. R. Oganov, Q. Zhu, Y. Tian, and H.-T. Wang, Phys. Rev. Lett. 112, 085502 (2014).

[19] F. Ma, Y. Jiao, G. Gao, Y. Gu, A. Bilic, Z. Chen, and A. Du, Nano Lett. 16, 3022 (2016).

[20] B. Feng, O. Sugino, R. Y. Liu, J. Zhang, R. Yukawa, M. Kawamura, T. Iimori, H. Kim, Y. Hasegawa, and H. Li et al., Phys. Rev. Lett. 118, 096401 (2017).

[21] M. Martinez-Canales, T. R. Galeev, A. I. Boldyrev, and C. J. Pickard, Phys. Rev. B 96, 195442 (2017).

[22] H. Wang, Q. Li, Y. Gao, F. Miao, X.-F. Zhou, and X. G. Wan, New J. Phys. 18, 073016 (2016).

[23] H. Zhou, Y. Cai, G. Zhang, and Y.-W. Zhang, npj 2D Mater. Appl. 1, 14 (2017). 
[24] E. S. Penev, A. Kutana, and B. I. Yakobson, Nano Lett. 16, 2522 (2016).

[25] M. Gao, Q.-Z. Li, X.-W. Yan, and J. Wang, Phys. Rev. B 95, 024505 (2017).

[26] Y. Zhao, S. Zeng, and J. Ni, Phys. Rev. B 93, 014502 (2016).

[27] C. Cheng, J.-T. Sun, H. Liu, H.-X. Fu, J. Zhang, X.-R. Chen, and S. Meng, 2D Mater. 4, 025032 (2017).

[28] Q. Gu, D. Xing, and J. Sun, Chin. Phys. Lett. 36, 097401 (2019).

[29] G. Csányi, C. J. Pickard, B. D. Simons, and R. J. Needs, Phys. Rev. B 75, 085432 (2007).

[30] K. Xia, H. Gao, C. Liu, J. Yuan, J. Sun, H.-T. Wang, and D. Xing, Sci. Bull. 63, 817 (2018).

[31] C. Liu, H. Gao, Y. Wang, R. J. Needs, C. J. Pickard, J. Sun, H.-T. Wang, and D. Xing, Nat. Phys. 15, 1065 (2019).

[32] K. Xia, J. Yuan, X. Zheng, C. Liu, H. Gao, Q. Wu, and J. Sun, J. Phys. Chem. Lett. 10, 6166 (2019).

[33] J. Wu, Z. Feng, J. Wang, Q. Chen, C. Ding, T. Chen, Z. Guo, J. Wen, Y. Shi, and D. Xing et al., Phys. Rev. B 100, 060103(R) (2019).

[34] C. J. Pickard and R. J. Needs, J. Phys.: Condens. Matter 23, 053201 (2011).

[35] S. J. Clark, M. D. Segall, C. J. Pickard, P. J. Hasnip, M. J. Probert, K. Refson, and M. C. Payne, Z. Kristallogr. 220, 567 (2005).

[36] G. Kresse and J. Furthmüller, Phys. Rev. B 54, 11169 (1996).

[37] J. P. Perdew, K. Burke, and M. Ernzerhof, Phys. Rev. Lett. 78, 1396 (1997).

[38] P. Giannozzi, S. Baroni, N. Bonini, M. Calandra, R. Car, C. Cavazzoni, D. Ceresoli, G. L. Chiarotti, M. Cococcioni, and I. Dabo et al., J. Phys.: Condens. Matter 21, 395502 (2009).
[39] S. Grimme, J. Antony, S. Ehrlich, and H. Krieg, J. Chem. Phys. 132, 154104 (2010).

[40] T. Thonhauser, V. R. Cooper, S. Li, A. Puzder, P. Hyldgaard, and D. C. Langreth, Phys. Rev. B 76, 125112 (2007).

[41] Y. Sun, J. Lv, Y. Xie, H. Liu, and Y. Ma, Phys. Rev. Lett. 123, 097001 (2019).

[42] Y. Wu, P. Lazic, G. Hautier, K. Persson, and G. Ceder, Energy Environ. Sci. 6, 157 (2013).

[43] Y. Hinuma, T. Hatakeyama, Y. Kumagai, L. A. Burton, H. Sato, Y. Muraba, S. Iimura, H. Hiramatsu, I. Tanaka, and H. Hosono et al., Nat. Commun. 7, 11962 (2016).

[44] D. Y. Kim, S. Stefanoski, O. O. Kurakevych, and T. A. Strobel, Nat. Mater. 14, 169 (2014).

[45] J. Wu, H. Gao, K. Xia, D. Xing, and J. Sun, Appl. Phys. Lett. 111, 173904 (2017).

[46] J. Song, H. Y. Song, Z. Wang, S. Lee, J. Y. Hwang, S. Y. Lee, J. Lee, D. Kim, K. H. Lee, and Y. Kim et al., Sci. Adv. 5, eaax0390 (2019).

[47] E. R. Johnson, S. Keinan, P. Mori-Sánchez, J. Contreras-García, A. J. Cohen, and W. Yang, J. Am. Chem. Soc. 132, 6498 (2010).

[48] P. B. Allen and R. C. Dynes, Phys. Rev. B 12, 905 (1975).

[49] J. Paier, R. Hirschl, M. Marsman, and G. Kresse, J. Chem. Phys. 122, 234102 (2005).

[50] A. A. Mostofi, J. R. Yates, Y.-S. Lee, I. Souza, D. Vanderbilt, and N. Marzari, Comput. Phys. Comm. 178, 685 (2008).

[51] F. Tran and P. Blaha, Phys. Rev. Lett. 102, 226401 (2009).

[52] A. R. Oganov and C. W. Glass, J. Chem. Phys. 124, 244704 (2006).

[53] A. Togo and I. Tanaka, Scr. Mater. 108, 1 (2015).

[54] A. Otero-de-la-Roza, E. R. Johnson, and V. Luaña, Comput. Phys. Comm. 185, 1007 (2014). 